\title{
Modelling and Analysis of the Hubble Diagram of 280 Type SNIa Supernovae and Gamma Ray Bursts Redshifts with Analytical and Empirical Redshift/Magnitude Functions
}

\author{
Laszlo A. Marosi \\ 67061 Ludwigshafen am Rhein, Germany \\ Email: LaszloMarosi@aol.com
}

Received 20 July 2016; accepted 28 August 2016; published 31 August 2016

Copyright (C) 2016 by author and Scientific Research Publishing Inc.

This work is licensed under the Creative Commons Attribution International License (CC BY).

http://creativecommons.org/licenses/by/4.0/

(c) (i) Open Access

\begin{abstract}
Based on an analysis of 280 Type SNIa supernovae and gamma-ray bursts redshifts in the range of $z=0.0104-8.1$ the Hubble diagram is shown to follow a strictly exponential slope predicting an exponentially expanding or static universe. At redshifts $>2$ - $3 \Lambda$ CDM models show a poor agreement with the observed data. Based on the results presented in this paper, the Hubble diagram test does not necessarily support the idea of expansion according to the big-bang concordance model.
\end{abstract}

Keywords

Magnitude, Redshift Data Fitting, Supernovae, Gamma Ray Bursts, Hubble Diagram, $\Lambda$ CDM Cosmological Model

\section{Introduction}

Based on an analysis of 280 Type SNIa supernovae and gamma ray bursts redshift (z) data Marosi [1] has shown that the best-fit function to represent the observational z/extinction-corrected distance moduli $(\mu)$ data set is the exponential equation $\mu=44.109769 \times z^{0.059883}$. Recently, a number of papers have appeared proposing different analytical formulae for describing the experimental $z / \mu$ relationship: Sorrell [2], Vigoureux, Vigoureux and Langlois [3] Traunmüller [4] and Churoux [5]. In this paper, observed $z / \mu$ data of 280 Type SNIa supernovae and 
gamma ray bursts in the range of $z=0.0104-8.1$ are compared to results calculated on the basis of these theoretically derived and empirical functions and the Lambda cold dark matter $(\Lambda \mathrm{CDM})$ model. The aim of this paper is to examine which of the above functions and models fits the observations more accurately. We expect that in the high RS range it should be possible to decide whether the Hubble diagram follows the distance/z relationship as predicted by the $\Lambda \mathrm{CDM}$ model, or the exponential tired light formula.

\section{Data Collection and Processing}

The $z / \mu$ data set consists of 171 gold-set data, Riess et al. [6], and 109 cosmology independent calibrated gamma-ray bursts (GRB) data consisting of 59 high-RS data (Hymnium data set) and 50 low RS GRBs obtained by Wei [7] from the 557 Union 2-compilation.

The following mathematical functions and cosmological models were used to perform a global fitting over the RS range of $z=0.0104-8.1$.

$$
\begin{gathered}
\mu=44.109 z^{0.0598} \text { (Marosi, 2014) } \\
\mu=25+5 \log \left(c / H_{0}\right)+5 \log ((z+1) \ln (z+1)) \text { (Vigoureux et al., 2014) } \\
\mu=2.5 \log \left((z+1) r_{u}^{2} z^{2}\right) \quad \text { (Churoux, 2015) }
\end{gathered}
$$

$\Lambda \mathrm{CDM}$ model with $H_{0}=62.5 \mathrm{~km} \cdot \mathrm{s}^{-1} \cdot \mathrm{Mpc}^{-1}, \Omega_{m}=0.286, \Omega_{\Lambda}=0.732$ and $k=0$

$\Lambda \mathrm{CDM}$ model with $H_{0}=72.6 \mathrm{~km} \cdot \mathrm{s}^{-1} \cdot \mathrm{Mpc}^{-1}, \Omega_{m}=0.286, \Omega_{\Lambda}=0.732$ and $k=0$

The $\mu$ values on basis of the $\Lambda \mathrm{CDM}$ models were calculated using:

$$
\mu=-5+5 \log D_{L},
$$

where $D_{L}(\mathrm{Pc})$ is the luminosity distance. The luminosity distances were calculated using the cosmological calculator described by Wright [8].

For preparing the linear $t_{S} / z$ Hubble diagram, using Equation (7) the fitted $z / \mu$ data were converted into the corresponding $t_{s} / z$ datasets.

The photon flight-time $t_{S}$ was calculated using:

$$
t_{s}=\frac{D_{c}}{c}=\frac{10 \frac{\mu+5}{5}}{(z+1) \times 3 \times 10^{10}} \times 3.085 \times 10^{18}
$$

In Equation (7) $t_{S}$ means the flight time of the photons (sec.) from the co-moving radial distance $D_{C}$ to the observer, $t_{S}=D_{C} / c$, which is proportional to the $D_{C}(\mathrm{Pc})$ that goes into the linear Hubble law.

In order to complete the fitted $z / \mu$ data set in the high RS range of $t_{S} \times 10^{-14}=6000-11000$, in addition to the measured RSs, using Equation (2), 41 equidistant $t_{S} / z$ data points were included into the Hubble diagram. The addition of the 41 additional data points is necessary to perform the $\sum \chi^{2}$-test between the best fit and the $\Lambda \mathrm{CDM}$ models because only few observations are available in this redshift range. The $t_{s} /$ RS values were calculated on the basis of Functions (2) and (5).

Excel and Excel Solver were used for data fitting, refinement, and data presentation.

\section{Results}

Figure 1 shows results of the $z / \mu$ data fitting with Functions (1)-(5).

It is easy to see by visual examination of Figure 1 that there are only minor differences between the fit curves calculated with Functions (1) and (2), whilst the $\Lambda \mathrm{CDM}$ model with $\mathrm{H}_{0}=72.6 \mathrm{~km} \mathrm{~s}^{-1} \mathrm{Mpc}^{-1}$ (bottom line in Figure 1) is slightly but perceptibly different.

The most reliable measure to quantify the differences between the individual fit curves turned out to be the $\sum \chi^{2}$-test. The goodness of fit indicators are shown in Table 1 .

The $\sum \chi^{2}$-test obviously favors the trend-lines obtained with Functions (1) and (2) and these two curves are practically congruent. On basis of the data presented in Table 1 the analytical best-fit Function (2) will be used as the best representation of the observed $z / \mu$ data in the following discussion.

Figure 2(a) and Figure 2(b) show Hubble diagrams calculated on basis of the Functions (2), (4) and (5). 


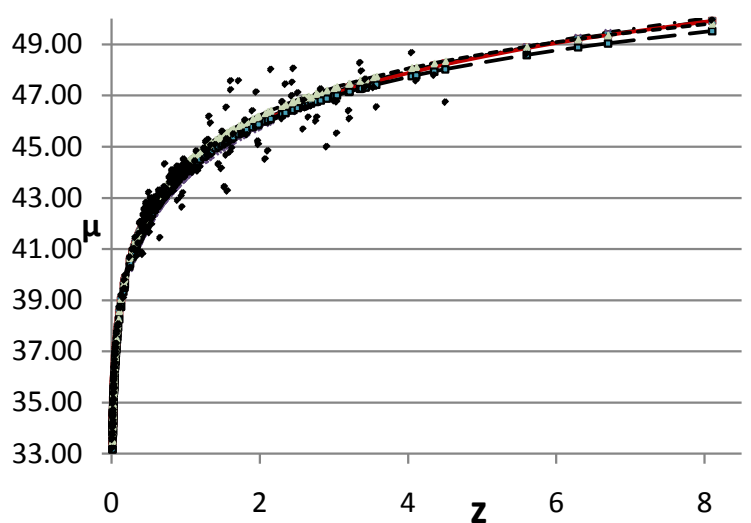

Figure 1. Squares: observed $z / \mu$ data; long dash-dot line: (2); solid line: (1); dash-dot line: (3); long dashed line; $\Lambda$ CDM, $\mathrm{H}_{0}=72.6 \mathrm{~km} \mathrm{~s}^{-1} \mathrm{Mpc}^{-1}$; short dashed line: $\Lambda \mathrm{CDM}, \mathrm{H}_{0}=62.5 \mathrm{~km} \mathrm{~s}^{-1} \mathrm{Mpc}^{-1}$.

Table 1. Goodness of fit indicators of the $z / \mu$ data trend-lines.

\begin{tabular}{ccccc}
\hline Function (F) & 1 & 2 & 3 & 4 \\
\hline$\sum \chi^{2}$ & 1.958865 & 1.96555 & 2.38869 & 2.163105 \\
$\mu_{o b s}-\mu_{F(1-5)}$ & & & 2.1582 \\
$\sum \chi^{2}$ & -0.34 & - & 17.78 & 9.57 \\
$\mathrm{~F} 2-\mathrm{F}(1 ; 3-5) \%$ & & & 8.9 \\
\hline
\end{tabular}

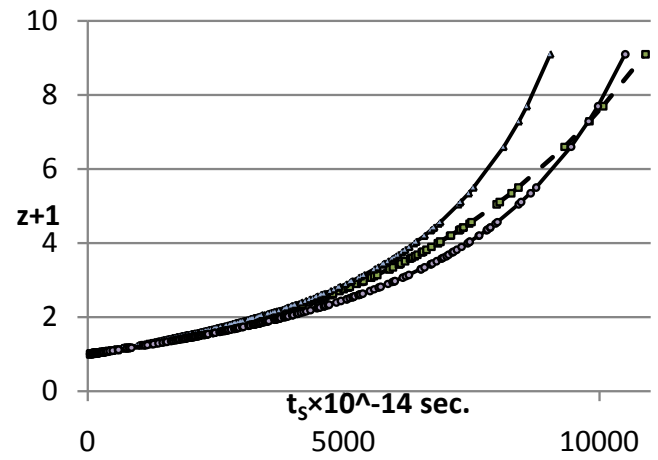

(a)

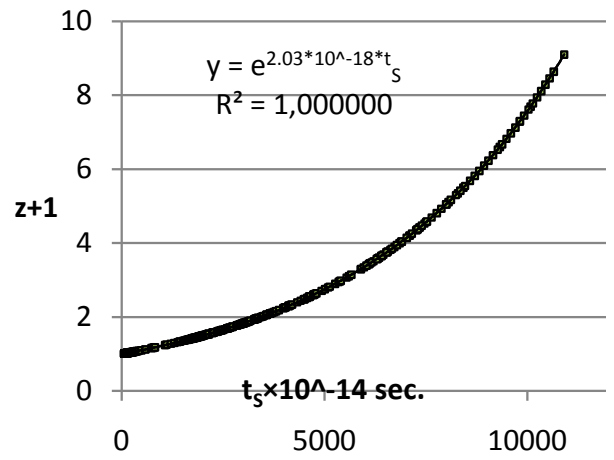

(b)

Figure 2. (a) Redshift of type Ia supernovae as a function of $t_{S}=D_{C} / c$. Squares (dashed line): $t_{S} / z$ data inferred from the potential best-fit curve of the observed $z / \mu$ diagram. Triangles: $t_{S} / z$ relationship derived from the $\Lambda \mathrm{CDM}$ model with $H_{0}=72.6 \mathrm{~km} \mathrm{~s}^{-1} \mathrm{Mpc}^{-1}$, Circles: $t_{S} / z$ relationship derived from the $\Lambda \mathrm{CDM}$ model with $H_{0}=62.5 \mathrm{~km} \mathrm{~s}^{-1} \mathrm{Mpc}^{-1}$; (b) squares: $t_{S} / z$ data inferred from the potential best-fit curve of the observed $z / \mu$ diagram, solid line: exponential trend-line (Excel).

\section{Discussion}

The most important result of the Hubble diagram test is that fitting the $t_{S} / z$ data with Function (2) and $H_{0}=62.5$ $\mathrm{km} \mathrm{s}^{-1} \mathrm{Mpc}^{-1}$ (dashed line in Figure 2(a)) leads exactly to the exponential function:

$$
Z+1=\mathrm{e}^{2.03^{*} 10^{-18} * t_{S}}
$$

as illustrated in Figure 2(b).

In spite of numerous correction factors and unknown constituents, dark matter (DM) and dark energy (DE), the $\Lambda \mathrm{CDM}$ models show a poor agreement with the observed data: as shown in Figure 2(a) the $\Lambda \mathrm{CDM}$ model with $H_{0}=62.5 \mathrm{~km} \mathrm{~s}^{-1} \mathrm{Mpc}^{-1}$ departs from the best-fit curve for $z+1<6.5$ to the bottom, for $z+1>6.5$ to the upper side of the trend-line. The deviations are of a systematic (nonstatistical) nature and, therefore, the model cannot reflect the observational exponential slope. 


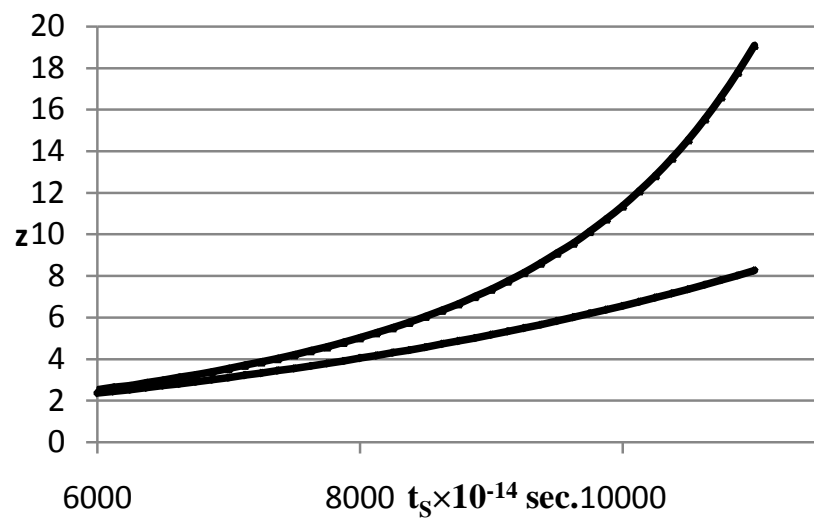

Figure 3. Bottom line: Calculated data points from the best-fit Function (2); upper line: calculated data points for the $t_{S} / z$ relationship derived from the $\Lambda \mathrm{CDM}$ model with $H_{0}=72.6 \mathrm{~km} \mathrm{~s}^{-1}$ $\mathrm{Mpc}^{-1}$. From [1].

In the range of $z>3$ the $\Lambda \mathrm{CDM}$ model with $H_{0}=72.6 \mathrm{~km} \mathrm{~s}^{-1} \mathrm{Mpc}^{-1}$ shows a sharp increase in slope and departs considerably from the observed exponential function. For performing the $\sum \chi^{2}$-test in the high RS range of $t_{S} \times 10^{-14}=6000-11000$ (Figure 3), using Equation (2), 41 calculated $t_{S} / z$ data points were included into the Hubble diagram. The $\sum \chi^{2}$-test leads to a statistical significance between the observed $t_{S} / \mu$ and the calculated $\Lambda \mathrm{CDM}$ data of $\mathrm{P}=0.053$, indicating that from the statistical point of view, the two models are essentially different.

\section{Conclusions}

The results presented in this paper have demonstrated that the $\Lambda$ CDM-model cannot fit the strictly exponential slope of the Hubble diagram in the entire RS range of $z=0.0104-8.1$, showing that the underlying theory is, at best, incomplete. A reconsideration of the $\Lambda$ CDM-model appears warranted.

The Hubble diagram test leads to the significant conclusion that either: (1) the universe expanded exponentially during the whole time of its expansion history (at least in the range of $z=0.0104-8.1$ ); or (2) the universe is static and the RS of spectral lines is caused by some as-yet unidentified mechanism. However, both of these models, the exponentially expanding and the static universe models have their own crucial problems; the discussion of them is not within the scope of this paper.

\section{References}

[1] Marosi, L.A. (2014) Hubble Diagram Test of 280 Supernovae Redshift Data. Journal of Modern Physics, 5, 29-33. http://dx.doi.org/10.4236/jmp.2014.51005

[2] Sorrell, W.H. (2009) Misconceptions about the Hubble Recession Law. Astrophysics and Space Science, 323, $205-211$. http://dx.doi.org/10.1007/s10509-009-0057-z

[3] Vigoureux, J.M., Vigoureux, B. and Langlois, M. (2014) An Analytical Expression for the Hubble Diagram of Supernovae and Gamma-Ray Bursts. arXiv:1411.3648v1.

[4] Traunmüller, H. (2014) From Magnitudes and Redshifts of Supernovae, Their Light-Curves, and Angular Sizes of Galaxies to a Tenable Cosmology. Astrophysics and Space Science, 350, 755-767. http://dx.doi.org/10.1007/s10509-013-1764-z

[5] Churoux, P. (2015) A New Interpretation of the Hubble Law. Journal of Modern Physics, 6, 1227-1232. http://dx.doi.org/10.4236/jmp.2015.69127

[6] Riess, A.G., Sirolger, A.G., Tonry, J., et al. (2004) Type Ia Supernova Discoveries at $z>1$ from the Hubble Space Telescope: Evidence for Past Deceleration and Constraints on Dark Energy Evolution. The Astrophysical Journal, 602, 665-687. http://dx.doi.org/10.1086/383612

[7] Wei, H. (2010) Observational Constraints on Cosmological Models with the Updated Long Gamma-Ray Bursts. Journal of Cosmology and Astroparticle Physics, 2010, Article ID: 020. http://dx.doi.org/10.1088/1475-7516/2010/08/020

[8] Wright, E.L. (2006) Cosmological Calculator. PASP, 188, 1711-1715, Last Modified 06-12-2014. 


\section{Submit or recommend next manuscript to SCIRP and we will provide best service for you:}

Accepting pre-submission inquiries through Email, Facebook, LinkedIn, Twitter, etc.

A wide selection of journals (inclusive of 9 subjects, more than 200 journals)

Providing 24-hour high-quality service

User-friendly online submission system

Fair and swift peer-review system

Efficient typesetting and proofreading procedure

Display of the result of downloads and visits, as well as the number of cited articles

Maximum dissemination of your research work

Submit your manuscript at: http://papersubmission.scirp.org/ 